\title{
Counting the Solutions of Presburger Equations without Enumerating Them *
}

\author{
Bernard Boigelot and Louis Latour \\ Institut Montefiore, B28 \\ Université de Liège \\ B-4000 Liège Sart-Tilman \\ Belgium \\ Phone: $+32-43662970$ \\ Fax: +32-43662984
}

\begin{abstract}
The Number Decision Diagram (NDD) has recently been introduced as a powerful representation system for sets of integer vectors. NDDs can notably be used for handling sets defined by arbitrary Presburger formulas, which makes them well suited for representing the set of reachable states of finite-state systems extended with unbounded integer variables. In this paper, we address the problem of counting the number of distinct elements in a set of numbers or, more generally, of vectors, represented by an NDD. We give an algorithm that is able to produce an exact count without enumerating explicitly the vectors, which makes it capable of handling very large sets. As an auxiliary result, we also develop an efficient projection method that allows to construct efficiently NDDs from quantified formulas, and thus makes it possible to apply our counting technique to sets specified by formulas. Our algorithms have been implemented in the verification tool LASH, and applied successfully to various counting problems.
\end{abstract}

Key words: Presburger arithmetic, automata, counting, symbolic representation systems.

ऋ This work was partially funded by a grant of the "Communauté française de Belgique - Direction de la recherche scientifique - Actions de recherche concertées", and by the European Commission (FET project ADVANCE, contract No IST-199929082).

Email address: $\{$ boigelot, latour\}@montefiore.ulg.ac.be (Bernard Boigelot and Louis Latour).

URL: http://www.montefiore.ulg.ac.be/ ${ }^{\sim}$ boigelot, latour\}/ (Bernard Boigelot and Louis Latour). 


\section{Introduction}

Presburger arithmetic [Pre29], i.e., the first-order additive theory of integers, is a powerful formalism for solving problems that involve integer variables. The manipulation of sets defined in Presburger arithmetic is central to many applications including integer programming problems [Sch86,PR96], compiler optimization techniques [Pug92], temporal database queries [KSW95], program analysis tools [FO97,SKR98] and model-checking [DK00,TBP99].

The most direct way of algorithmically handling Presburger-definable sets consists in manipulating Presburger formulas explicitly. This approach has been successfully implemented in the Omega package [Pug92], which is probably the most widely used Presburger tool at the present time. Unfortunately, formulabased representations suffer from a serious drawback: They lack canonicity, which implies that a set with a simple structure may in some instances be represented by a complex formula; this notably happens when the set is obtained as the result of a lengthy sequence of operations. Moreover, the absence of a canonical representation hinders the efficient implementation of decision procedures that are essential to most applications, such as testing whether two sets are equal.

In order to alleviate these problems, an alternative representation of Presburger-definable sets has been developed, based on finite-state automata. The Number Decision Diagram (NDD) [WB95,Boi99] is, sketchily, a finite-state machine recognizing the encodings of the integer vectors belonging to the set that it represents. Its main advantages are that most of the usual set-theory operators can be applied to the represented sets by simply carrying out the corresponding tasks on the languages accepted by the underlying automata, and that a canonical representation of a set can easily be obtained by determinizing and minimizing its finite-state representations. Among its applications, the NDD has made it possible to develop a tool for automatically computing the set of reachable states of programs using unbounded integer variables [LAS].

The problem of counting how many elements belong to a Presburger set has been solved for formula-based representations of Presburger sets [Pug94]. This problem has interesting applications related to program analysis and verification. First, it enables one to quantify precisely and to improve the performance of some systems. In particular, by defining Presburger formulas whose solutions correspond to the memory locations touched by a loop and the flops executed by a loop, one can estimate the amount of resources consume by code fragments and improve the load balancing in a multiprocessor environment [TF92]. Furthermore, counting the number of reachable data values at selected control locations makes it possible to detect quickly some inconsistencies between different releases of a program, without requiring to write down 
explicit properties to be checked. For instance, it can promptly alert the developer, although without any guarantee of always catching such errors, that a local modification had an unwanted influence on some remote part of the program. Finally, studying the evolution of the number of reachable states with respect to the value of system parameters can also help to detect unsuspected errors.

The main goal of this paper is to present a method for counting exactly and efficiently the number of elements belonging to a Presburger-definable set represented by an NDD. Intuitively, our approach is based on the idea that one can easily compute the number of distinct paths in a directed acyclic graph without enumerating them. The actual algorithm is however more intricate, due to the fact that there is not a one-to-one relationship between the vectors belonging to a set and the accepting paths of NDD representing the set.

In order to apply our counting technique to the set of solutions of a given Presburger formula, one needs first to build an NDD from that formula. This problem has been solved in [BHMV94,BC96,Boi99], but only in the form of a construction algorithm that presents a systematic exponential cost in the number of variables that appear in the formula. As an auxiliary contribution of this paper, we describe an improved algorithm for handling the problematic projection operation. The resulting construction procedure has been implemented and successfully applied to problems involving a large number of variables.

\section{Basic notions}

Let us first show how finite-state machines can represent sets of integer vectors. The main idea consists of establishing a mapping between vectors and words. Our encoding scheme for vectors is based on the positional notation for numbers in a base $r>1$, according to which an encoding of a positive integer $z$ is a word $a_{p-1} a_{p-2} \cdots a_{1} a_{0}$ such that each digit $a_{i}$ belongs to the finite alphabet $\{0,1, \ldots, r-1\}$ and $z=\sum_{i=0}^{p-1} a_{i} r^{i}$. An encoding of a negative number $z$ is the last $p$ digits of any encoding of its $r^{\prime}$ s complement $r^{p}+z$. The number $p$ of digits is not fixed, but must be large enough for the condition $-r^{p-1} \leq z<r^{p-1}$ to hold. As a result, the first digit of the encodings is 0 for positive numbers and $r-1$ for negative ones, hence that digit is referred to as the sign digit of the encodings.

In order to encode a vector $\vec{v}=\left(v_{1}, v_{2}, \ldots, v_{n}\right)$, one simply reads repeatedly and in turn one digit from the encodings of all its components, under the additional restriction that these encodings must share the same length. In other words, an encoding of $\vec{v}$ is a word $d_{p-1,1} d_{p-1,2} \ldots d_{p-1, n} d_{p-2,1} d_{p-2,2} \ldots d_{p-2, n} \ldots$ $\ldots d_{0,1} d_{0,2} \ldots d_{0, n}$ such that for every $i \in\{1, \ldots, n\}, d_{p-1, i} d_{p-2, i} \ldots d_{0, i}$ is an 
encoding of $v_{i}$. An encoding of a vector of dimension $n$ has thus $n$ sign digits - each associated to one vector component - the group of which forms a sign header. Two encodings of the same vector can only differ in the number of times that their sign header is repeated.

Let $S \subseteq \mathbb{Z}^{n}$ be a set of integer vectors. If the language $L(S)$ containing all the encodings of all the vectors in $S$ is regular, then any finite-state automaton accepting $L(S)$ is a Number Decision Diagram (NDD) representing $S$. It is worth noticing that, according to this definition, not all automata defined over the alphabet $\{0,1, \ldots, r-1\}$ are valid NDDs. Indeed, an NDD must accept only valid encodings of vectors sharing all the same dimension, and must accept all the encodings of each vector that it recognizes. Note that the vector encoding scheme that we use here is slightly different from the one proposed in [BHMV94,Boi99], in which the digits related to all the vector components are read simultaneously rather than successively. It is easy to see that both representation methods are equivalent from the theoretical point of view. The advantage of our present choice is that it produces considerably more compact finite-state representations. For instance, a deterministic and minimal NDD representing $\mathbb{Z}^{n}$ is of size $O\left(2^{n}\right)$ if the component digits are read simultaneously, which limits the practical use of that approach to small values of $n$. On the other hand, the encoding scheme used in this paper yields an automaton of size $O(n)$.

It is known for a long time [Cob69,Sem77] that the sets that can be represented by finite-state automata in every base $r>1$ are exactly those that are definable in Presburger arithmetic, i.e., the first-order theory $\langle\mathbb{Z},+, \leq\rangle$. One direction of the proof of this result is constructive, and translates into an algorithm for constructing an NDD representing an arbitrary Presburger formula [BHMV94,BC96,Boi99]. Sketchily, the idea is to start from elementary NDDs corresponding to the formula atoms, and to combine the NDDs by means of set operators and quantification. It can be easily shown that computing the union, intersection, difference or Cartesian product of two sets represented by NDDs is equivalent to carrying out similar operations on the languages accepted by the underlying automata. Quantifying existentially a set with respect to a vector component, which amounts to projecting this set along this component, is more complex. We discuss this problem in the next section.

At this point, one could wonder why we did not opt for defining NDDs as automata accepting only one encoding (for instance the shortest one) of each vector, and encoding negative numbers as their sign followed by the encoding of their absolute value. It turns out that the former choice substantially complicates the essential manipulation algorithms such as computing the Cartesian product or the difference of two sets (in this case, the problem is that those operations do not reduce to carrying out similar operations over the languages 


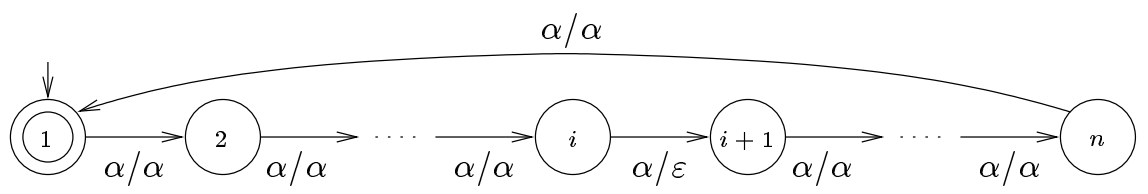

For all transitions, $\alpha \in\{0, \ldots, r-1\}$. The symbol $\varepsilon$ denotes the empty word.

Fig. 1. Projection transducer.

accepted by the automata). The latter choice leads to significantly larger representations for atomic formulas such as linear equations or inequations. On the other hand, our present choices lead to simple manipulation algorithms, with the only exceptions of projection and counting, which are addressed in the following sections.

\section{Projecting NDDs}

The projection problem can be stated in the following way. Given an NDD $\mathcal{A}$ representing a set $S \subseteq \mathbb{Z}^{n}$, with $n>0$, and a component number $i \in$ $\{1, \ldots, n\}$, construct an NDD $\mathcal{A}^{\prime}$ representing the set

$$
\exists_{i} S=\left\{\left(v_{1}, \ldots, v_{i-1}, v_{i+1}, \ldots, v_{n}\right) \mid\left(v_{1}, \ldots, v_{n}\right) \in S\right\} .
$$

For every accepting path of $\mathcal{A}$, there must exist a matching path of $\mathcal{A}^{\prime}$, the label of which contains digits corresponding to all the vector components but the $i$-th. Thus, one could be tempted to compute $\mathcal{A}^{\prime}$ as the direct result of applying to $\mathcal{A}$ the transducer depicted at Figure 1.

Unfortunately, this method produces an automaton $\left.\mathcal{A}\right|_{\neq i}$ that, even though it accepts valid encodings of all the elements of $\exists_{i} S$, is generally not an NDD. Indeed, for some vectors, the automaton may only recognize their encodings if they are of sufficient length. For example, the method applied to the NDD representing $\exists_{1}\{(4,1)\}$ would generate an automaton whose smallest accepted word is "0001" which is not the smallest valid encoding of the number 1, i.e "01". In order to build $\mathcal{A}^{\prime}$ from $\left.\mathcal{A}\right|_{\neq i}$, one thus has to transform $\left.\mathcal{A}\right|_{\neq i}$ such that it also accepts the shorter encodings of the vectors of the set $\exists_{i} S$.

As already mentioned, two encodings of the same vector only differ in the number of times that their sign header is repeated. We can thus restate the projection problem in the following way: Given a finite-state automaton $\mathcal{A}_{1}$ over alphabet $\Sigma$ accepting the language $L_{1}$, and a dimension $n \geq 0$, construct an automaton $\mathcal{A}_{2}$ accepting $L_{2}=\left\{u^{i} w \mid u \in\{0, r-1\}^{n} \wedge w \in \Sigma^{*} \wedge i>\right.$ $\left.0 \wedge \exists k\left(k \geq i \wedge u^{k} w \in L_{1}\right)\right\}$. 
In [Boi99], this problem is solved by considering explicitly every potential value $u$ of the sign header. Then, for each $u$, explore $\mathcal{A}_{1}$ in order to know which states can be reached by a prefix of the form $u^{i}$, with $i>0$ and make each of these states reachable after reading a single occurrence of $u$, which can be done by a simple construction. Although satisfactory from a theoretical point of view, this solution exhibits a systematic cost in $O\left(2^{n}\right)$ which limits its practical use to problems with a very small vector dimension.

The main idea behind our improved solution consists of handling simultaneously sign headers that cannot be distinguished from each other by the automaton $\mathcal{A}_{1}$, i.e., sign headers $u_{1}, u_{2} \in\{0, r-1\}^{n}$ such that for every $k>0$, reading $u_{1}^{k}$ leads to the same automaton states as reading $u_{2}^{k}$. For simplicity, we assume $\mathcal{A}_{1}$ to be deterministic ${ }^{1}$.

Our algorithm proceeds as follows. First, it extracts from $\mathcal{A}_{1}$ a prefix automaton $\mathcal{A}_{P}$ that reads only the first $n$ symbols of words and associates one distinct end state to each group of undistinguished sign headers. Each end state of $\mathcal{A}_{P}$ is then matched to all the states of $\mathcal{A}_{1}$ that can be reached after reading the corresponding sign headers any number of times. At every time during this operation when one detects two sign headers that are not yet distinguished in $\mathcal{A}_{P}$ but that lead to different states of $\mathcal{A}_{1}$, one refines the prefix automaton $\mathcal{A}_{P}$ so as to associate different end states to these headers. Finally, the automaton $\mathcal{A}_{2}$ is constructed such that following one of its accepting paths amounts to reading $n$ symbols in $\mathcal{A}_{P}$, which reaches one of its end states $s$, and then following an accepting path of $\mathcal{A}_{1}$ starting from a state matched to $s$.

The algorithm is formally described in Appendix A. Its worst-case time complexity $O\left(2^{n}\right)$ is not less than that of the simple solution [Boi99] outlined at the beginning of this section. However, in the context of state-space exploration applications, is has been observed that it succeeds most of the time, if not always, to avoid the exponential blowup experienced with the latter approach.

\section{Counting elements of NDDs}

We now address the problem of counting the number of vectors that belong to a set $S$ represented by an NDD $\mathcal{A}$. Our solution proceeds in two steps: First, we check whether $S$ is finite or infinite and, in the former case, we transform $\mathcal{A}$

$\overline{1}$ This is not problematic in practical applications, since the cost of determinizing an automaton built from an arithmetic formula is often moderate [WB00] although the worst case complexity is $O\left(2^{\mid} Q \mid\right)$ where $|Q|$ is the number of states of the automaton. 
into a deterministic automaton $\mathcal{A}^{\prime}$ that accepts exactly one encoding of each vector that belongs to $S$. Second, we count the number of distinct accepting paths in $\mathcal{A}^{\prime}$.

\subsection{Transformation step}

Let $\mathcal{A}$ be a deterministic and minimal NDD representing the set $S \subseteq \mathbb{Z}^{n}$. If $S$ is not empty, then the language accepted by $\mathcal{A}$ is infinite, hence the transition graph of this automaton contains cycles. In order to check whether $S$ is finite or not, we thus have to determine if these cycles are always followed when reading different encodings of the same vectors, or if they can be iterated in order to recognize an infinite number of distinct vectors.

Assume that $\mathcal{A}$ does not contain unnecessary states, i.e., that all its states are reachable and that there is at least one accepting path originating in each state. We can classify the cycles in $\mathcal{A}$ into three groups:

- A sign loop is a cycle that can only be followed while reading the sign header of an encoding, or a repetition of that sign header;

- An inflating loop is a cycle that can never be followed while reading the sign header of an encoding or one of its repetitions;

- A mixed loop is a cycle that is neither a sign nor an inflating loop.

If $\mathcal{A}$ has at least one inflating or mixed loop, then its transition graph admits an accepting path that follows the corresponding cycle while not reading a repetition of a sign header. By iterating this cycle, one thus gets an infinite number of distinct vectors, which results in $S$ being infinite. The problem of checking if $S$ is infinite thus reduces to determining whether $\mathcal{A}$ has at least one non-sign (i.e., inflating or mixed) loop ${ }^{2}$. Thanks to the following result, this check can be carried out by inspecting the transition graph of $\mathcal{A}$ without paying attention to the transition labels.

Theorem 1 Assume that $\mathcal{A}$ is a deterministic and minimal NDD. A cycle $\lambda$ of $\mathcal{A}$ is a sign loop if and only if it can only be reached by one path (not containing any occurrence of that cycle).

PROOF. Since $\mathcal{A}$ is an NDD, it can only accept words whose length is a

2 An example of a non-trivial instance of this problem can be obtained by building the minimal deterministic NDD representing the set $\left\{(x, y) \in \mathbb{Z}^{2} \mid x+y \leq 0 \wedge x \geq\right.$ $0\}$. 
multiple of the vector dimension $n$. The length of $\lambda$ is thus a multiple of $n$.

- Assume $\lambda$ is reachable by only one path $\pi$. Let $u \in\{0, r-1\}^{n}$ be the sign header that is read while following the $n$ first transitions of the path $\pi \lambda$, and let $s$ and $s^{\prime}$ be the states of $\mathcal{A}$ respectively reached after reading the words $u$ and $u u$ (starting from the initial state).

Since $\mathcal{A}$ accepts all the encodings of the vectors in $S$, it accepts, for every $w \in\{0,1, \ldots, r-1\}^{*}$, the word $u w$ if and only if it accepts the word uuw. It follows that the languages accepted from the states $s$ and $s^{\prime}$ are identical which implies, since $\mathcal{A}$ is minimal, that $s=s^{\prime}$.

Therefore, $\lambda$ can only be visited while reading the sign header $u$ or one of its repetitions, and is thus a sign loop.

- Assume $\lambda$ is reachable by at least two paths $\pi_{1}$ and $\pi_{2}$. Let $k n$, with $k \in \mathbb{N}$, be the length of $\lambda$. Since $\mathcal{A}$ only accepts words whose length is a multiple of $n$, there are exactly $k$ states $s_{1}, s_{2}, \ldots, s_{k}$ that are reachable in $\lambda$ from the initial state of $\mathcal{A}$ after following a multiple of $n$ transitions.

If the words read by following $\lambda$ from $s_{1}$ to $s_{2}$, from $s_{2}$ to $s_{3}, \ldots$, and from $s_{k}$ to $s_{1}$ are not all identical, then $\lambda$ is not a sign loop.

Otherwise, let $u^{k}$, with $u \in\{0,1, \ldots, r-1\}^{n}$, be the label of $\lambda$. Since $\mathcal{A}$ is deterministic, at least one of the blocks of $n$ consecutive digits read while following $\pi_{1}$ or $\pi_{2}$ up to reaching $\lambda$ differs from $u$. Thus, $\lambda$ can be visited while not reading a repetition of a sign header, and is not a sign loop.

Provided that $\mathcal{A}$ has only sign loops, it can easily be transformed into an automaton $\mathcal{A}^{\prime}$ that accepts exactly one encoding of each vector in $S$ by performing a depth-first search in its transition graph. During the search, one removes for each detected cycle the transition that gets back to a state that has already been visited in the current exploration path. This operation does not influence the set of vectors recognized by the automaton, since the removed transitions can only be followed in $\mathcal{A}$ while reading a repeated occurrence of a sign header.

An algorithm that combines the classification of cycles with the transformation of $\mathcal{A}$ into $\mathcal{A}^{\prime}$ is given in Appendix B. Since each state of $\mathcal{A}$ needs to be visited at most once, the time and space costs of this algorithm - if suitably implemented - are linear in the number of states of $\mathcal{A}^{3}$.

3 In the algorithm provided in Appendix $\mathrm{B}$, given an automaton $\mathcal{A}\left(\Sigma, Q, s^{(0)}, \Delta, F\right)$, the subroutine explore() is called at most $|Q|$ times and all tests and instructions except the recursive call to the subroutine can be performed in constant time. 


\subsection{Counting step}

If $S$ is finite, then the transition graph of the automaton $\mathcal{A}^{\prime}$ produced by the algorithm given in the previous section is acyclic. The number of vectors in $S$ corresponds to the number of accepting paths originating in the initial state of $\mathcal{A}^{\prime}$.

For each state $s$ of $\mathcal{A}^{\prime}$, let $N(s)$ denote the number of paths of $\mathcal{A}^{\prime}$ that start in $s$ and end in an accepting state. Each of these paths either leaves $s$ by one of its outgoing transitions, or has a zero length (in which case $s$ is accepting). Thus, we have at each state $s$

$$
N(s)=\sum_{\left(s, d, s^{\prime}\right) \in \Delta} N\left(s^{\prime}\right)+a c c(s)
$$

where $\operatorname{acc}(s)$ is equal to 1 if $s$ is accepting, and to 0 otherwise.

Thanks to this rule, the value of $N(s)$ can easily be propagated from the states that have no successors to the initial state of $\mathcal{A}^{\prime}$, following the transitions backwards. The number of additions that have to be performed is linear in the number of states of $\mathcal{A}^{\prime}$.

\section{Example of use}

The projection and counting algorithms presented in Sections 3 and 4 have been implemented in the verification tool LASH [LAS], whose main purpose is to compute exactly the set of reachable configurations of systems with finite control and unbounded data. In short, this tool handles finite and infinite sets of configurations by means of finite-state representations suited for the corresponding data domains, and relies on meta-transitions, which capture the effect of control loops, for exploring infinite state spaces in finite time. A description of the main techniques implemented in LASH is given in [Boi99].

In the context of this paper, we focus on systems based on unbounded integer variables, for which the set representation system used by LASH is the NDD. Our present results thus make it possible to count precisely the number of reachable system configurations that belong to a set computed by LASH.

Let us now describe an example of a state-space exploration experiment featuring the counting algorithm. We consider the simple lift controller originally presented in [Val89]. This system is composed of two processes modeling a lift panel and its motor actuator, communicating with each other by means 
Table 1

\begin{tabular}{|r|r|r|r|}
\hline$N$ & NDD states & Configurations & Time (s) \\
\hline \hline 10 & 852 & 930 & 25 \\
\hline 100 & 1782 & 99300 & 65 \\
\hline 1000 & 2684 & 9993000 & 101 \\
\hline 10000 & 3832 & 999930000 & 153 \\
\hline 100000 & 4770 & 99999300000 & 196 \\
\hline 1000000 & 5666 & 9999993000000 & 242 \\
\hline
\end{tabular}

Number of reachable configurations w.r.t. $N$.

of shared integer variables. A parameter $N$, whose value is either fixed in the model or left undetermined, defines the number of floors of the building. In the former case, one observes that the amount of time and of memory needed by LASH in order to compute the set of reachable configurations ${ }^{4}$ grows only logarithmically in $N$, despite the fact that the number of elements in this set is clearly at least $O\left(N^{2}\right)$. (Indeed, the behavior of the lift is controlled by two main variables modeling the current and the target floors, which are able to take any pair of values in $\{1, \ldots, N\}^{2}$.) Our simple experiment has two goals: Studying precisely the evolution of the number of reachable configurations with respect to increasing values of $N$, and evaluating the amount of acceleration induced by meta-transitions in the state-space exploration process.

The results are summarized in Tables 1 and 2. The former table gives, for several values of $N$, the size (in terms of automaton states) of the finite-state representation of the set of reachable configurations, the exact number of these configurations, and the total time needed to perform the exploration. These results clearly show an evolution in $O\left(N^{2}\right)$, as expected. It is worth mentioning that, thanks to the fact that the cost of our counting algorithm is linear in the size of NDDs, its execution time (including the classification of loops) was negligible with respect to that of the exploration.

The latter table shows, for $N=10^{9}$, the evolution of the number of configurations reached after the successive steps of the exploration algorithm. Roughly

${ }^{4}$ Practically, the reachable configurations are computed as follows. First we compute the transition fonction for the system. This function takes a set of configurations as input and generates the set of configurations reachable from the input set in one-step. Then, we apply this transition fonction recusively to the initial configuration until we reach a fixpoint. In our example, the Presburger formula corresponding to the transition fonction is an union of about 100 clauses and has 14 variables of which 9 are quantified existentially. We need to apply 11 times the transition fonction to explore the system completely. 
Table 2

\begin{tabular}{|r|r|r|}
\hline Step & NDD states & Configurations \\
\hline \hline 1 & 638 & 3 \\
\hline 2 & 1044 & 1000000003 \\
\hline 3 & 1461 & 3999999999 \\
\hline 4 & 2709 & 500000005499999997 \\
\hline 5 & 4596 & 1500000006499999995 \\
\hline 6 & 6409 & 3500000004499999994 \\
\hline 7 & 7020 & 6499999997499999999 \\
\hline 8 & 7808 & 7999999995000000000 \\
\hline 9 & 8655 & 8999999994000000000 \\
\hline 10 & 8658 & 9499999993500000000 \\
\hline 11 & 8663 & 9999999993000000000 \\
\hline
\end{tabular}

Number of reached configurations w.r.t. exploration steps.

speaking, the states are explored in a breadth-first fashion, starting from the initial configuration and following transitions as well as meta-transitions, until a fixpoint is detected. In the present case, the impact of meta-transitions on the number of reached states is clearly visible at Steps 2 and 4 in the table.

\section{Conclusions and comparison with other work}

The main contribution of this paper is to provide an algorithm for counting the number of elements in a set represented by an NDD. As an auxiliary result, we also present an improved projection algorithm that makes it possible to build efficiently an NDD representing the set of solutions of a Presburger formula. Our algorithms have been implemented in the tool LASH.

The problem of counting the number of solutions of a Presburger equation has already been addressed in [Pug94], following a formula-based approach. More precisely, that solution proceeds by decomposing the original formula into an union of disjoint convex sums, each of them being a conjunction of linear inequalities. Then, all variables but one are projected out successively, by splintering the sums in such a way that the eliminated variables have one single and one upper bound. This eventually yields a finite union of simple formulas, on which the counting can be carried out by simple rules. 
The main difference between this solution and ours is that, compared to the general problem of determining whether a Presburger formula is satisfiable, counting using a formula-based method incurs a significant additional cost. On the other hand, the automata-based counting method has a negligible practical impact on the total execution time once an NDD has been constructed. Our method is thus efficient in all the cases for which an NDD can be computed quickly, which, as it has been observed in [BC96,WB00], happens mainly when the coefficients of the variables are kept small. In addition, since automata can be determinized and minimized after each manipulation, NDDs are especially suited for representing the results of complex sequences of operations producing simple sets, as in most state-space exploration applications. The main restriction of our approach is that it cannot be generalized in a simple way to the more complex counting problems, such as summing polynomials over Presburger-definable sets, that are addressed in [Pug94].

\section{References}

[BC96] A. Boudet and H. Comon. Diophantine equations, Presburger arithmetic and finite automata. In Proceedings of CAAP'96, number 1059 in Lecture Notes in Computer Science, pages 30-43. Springer-Verlag, 1996.

[BHMV94] V. Bruyère, G. Hansel, C. Michaux, and R. Villemaire. Logic and p-recognizable sets of integers. Bulletin of the Belgian Mathematical Society, 1(2):191-238, March 1994.

[Boi99] B. Boigelot. Symbolic Methods for Exploring Infinite State Spaces. Collection des publications de la Faculté des Sciences Appliquées de l'Université de Liège, Liège, Belgium, 1999.

[Cob69] A. Cobham. On the base-dependence of sets of numbers recognizable by finite automata. Mathematical Systems Theory, 3:186-192, 1969.

[DK00] Z. Dang and R.A Kemmerer. Using the astral symbolic model checker as a specification debugger: Three approximation techniques. In E. M. Clarke and R. P. Kurshan, editors, Proceedings of the 22nd International Conference on Software Engineering (ICSE 2000). IEEE Press, IEEE Press, 2000.

[FO97] L. Fribourg and H. Olsén. Proving safety properties of infinite state systems by compilation into Presburger arithmetic. In Proceedings of CONCUR'97, volume 1243, pages 213-227, Warsaw, Poland, July 1997. Springer-Verlag.

[KSW95] F. Kabanza, J.-M. Stevenne, and P. Wolper. Handling infinite temporal data. Journal of computer and System Sciences, 51(1):3-17, 1995.

[LAS] The Liège Automata-based Symbolic Handler (LASH). Available at http://www . montefiore.ulg.ac .be/ boigelot/research/lash/. 
[PR96] M. Padberg and M. Rijal. Location, Scheduling, Design and Integer Programming. Kluwer Academic Publishers, Massachusetts, 1996.

[Pre29] M. Presburger. Über die Volständigkeit eines gewissen Systems der Arithmetik ganzer Zahlen, in welchem die Addition als einzige Operation hervortritt. In Comptes Rendus du Premier Congrès des Mathématiciens des Pays Slaves, pages 92-101, Warsaw, Poland, 1929.

[Pug92] W. Pugh. The Omega Test: A fast and practical integer programming algorithm for dependence analysis. Communications of the $A C M$, pages 102-114, August 1992.

[Pug94] W. Pugh. Counting solutions to Presburger formulas: How and why. SIGPLAN, 94-6/94:121-134, 1994.

[Sch86] A. Schrijver. Theory of Linear and Integer Programming. John Wiley \& sons, Chichester, 1986.

[Sem77] A. L. Semenov. Presburgerness of predicates regular in two number systems. Siberian Mathematical Journal, 18:289-299, 1977.

[SKR98] T. R. Shiple, J. H. Kukula, and R. K. Ranjan. A comparison of Presburger engines for EFSM reachability. In Proceedings of the 10th Intl. Conf. on Computer-Aided Verification, volume 1427 of Lecture Notes in Computer Science, pages 280-292, Vancouver, June/July 1998. Springer-Verlag.

[TBP99] R. Gerber T. Bultan and W. Pugh. Model-checking concurrent systems with unbounded integer variables: symbolic representations, approximations, and experimental results. ACM Transactions on Programming Languages and Systems (TOPLAS), 21(4):747-789, July 1999.

[TF92] N. Tawbi and P. Feautrier. Processor allocation and loop scheduling on multiprocessor computers. In Proceedings of the 6th international conference on Supercomputing, pages 63-71. ACM Press, 1992.

[Val89] A. Valmari. State space generation with induction. In Proceedings of the SCAI'89, pages 99-115, Tampere, Finland, June 1989.

[WB95] P. Wolper and B. Boigelot. An automata-theoretic approach to Presburger arithmetic constraints. In Proceedings of Static Analysis Symposium, volume 983 of Lecture Notes in Computer Science, pages 21-32, Glasgow, September 1995. Springer-Verlag.

[WB00] P. Wolper and B. Boigelot. On the construction of automata from linear arithmetic constraints. In Proc. 6th International Conference on Tools and Algorithms for the Construction and Analysis of Systems, volume 1785 of Lecture Notes in Computer Science, pages 1-19, Berlin, March 2000. Springer-Verlag. 


\section{A Projection algorithm}

Let $\left(\Sigma, Q, s^{(0)}, \Delta, F\right)$ be the deterministic automaton $\mathcal{A}_{1}$, where $\Sigma$ is the alphabet $\{0, \ldots, r-1\}, Q$ is a finite set of states, $s^{(0)} \in Q$ is the initial state, $\Delta \subseteq Q \times \Sigma \times Q$ is the transition relation, and $F \subseteq Q$ is a set of accepting states.

(1) Let $\mathcal{A}_{P}=\left(\Sigma, Q_{P}, s_{P}^{(0)}, \Delta_{P}, F_{P}\right)$, with $s_{P}^{(0)}=\left(s^{(0)}, 0\right), Q_{P}=\left\{s_{P}^{(0)}\right\}$, and both of $\Delta_{P}$ and $F_{P}$ are empty. Each state $(s, i)$ of $\mathcal{A}_{P}$ will be composed of a state $s$ of $\mathcal{A}_{1}$ and an index $i$ ranging from 0 to $n$. The index $n$ corresponds to the end states of $\mathcal{A}_{P}$.

(2) The array matches[s] will associate each end states of $\mathcal{A}_{P}$ to the subset of $Q$ such that

(3) For $i=1, \ldots, n$ and for each $\left(s, \alpha, s^{\prime}\right) \in \Delta$ such that $(s, i-1) \in Q_{P}$, add $\left(s^{\prime}, i\right)$ to $Q_{P}$ and $\left((s, i-1), \alpha,\left(s^{\prime}, i\right)\right)$ to $\Delta_{P}$.

(4) For each $s \in Q$ such that $(s, n) \in Q_{P}$, let matches $[(s, n)]=\{s\}$.

(5) Let remaining $=\left\{(s, s) \mid(s, n) \in Q_{P}\right\}$.

(6) For each $\left(s, s^{\prime}\right) \in$ remaining:

- If there do not exist $s^{\prime \prime} \in Q \backslash$ matches $[(s, n)]$ and $u \in \Sigma^{n}$ such that $\left(s_{P}^{(0)}, u,(s, n)\right) \in \Delta_{P}^{*}$ and $\left(s^{\prime}, u, s^{\prime \prime}\right) \in \Delta^{*}$, then remove $\left(s, s^{\prime}\right)$ from remaining .

- If there exists $s^{\prime \prime} \in Q \backslash$ matches $[(s, n)]$ such that for every $u \in \Sigma^{n}$ for which $\left(s_{P}^{(0)}, u,(s, n)\right) \in \Delta_{P}^{*},\left(s^{\prime}, u, s^{\prime \prime}\right) \in \Delta^{*}$, then add $s^{\prime \prime}$ to the set matches $[(s, n)]$, add $\left(s, s^{\prime \prime}\right)$ to remaining, and remove $\left(s, s^{\prime}\right)$ from remaining.

- Otherwise, find $u, u^{\prime} \in \Sigma^{n}$ such that $\left(s_{P}^{(0)}, u,(s, n)\right) \in \Delta_{P}^{*},\left(s_{P}^{(0)}, u^{\prime},(s, n)\right)$ $\in \Delta_{P}^{*}$ and either

- there exist $s^{\prime \prime}, s^{\prime \prime \prime} \in Q, s^{\prime \prime} \neq s^{\prime \prime \prime}$, such that $\left(s^{\prime}, u, s^{\prime \prime}\right) \in \Delta^{*}$ and $\left(s^{\prime}, u^{\prime}, s^{\prime \prime \prime}\right) \in \Delta^{*}$, or

- there exists $s^{\prime \prime} \in Q$ such that $\left(s^{\prime}, u, s^{\prime \prime}\right) \in \Delta^{*}$ but no $s^{\prime \prime \prime} \in Q$ such that $\left(s^{\prime}, u^{\prime}, s^{\prime \prime \prime}\right) \in \Delta^{*}$,

then refine $\mathcal{A}_{P}$ with respect to the state $s^{\prime}$ and the headers $u$ and $u^{\prime}$ (this operation will be described separately).

(7) Let $\mathcal{A}_{2}=\left(\Sigma, Q_{2}, s_{2}^{(0)}, \Delta_{2}, F_{2}\right)$, with $Q_{2}=Q \cup Q_{P}, s_{2}^{(0)}=s_{P}^{(0)}, \Delta_{2}=$ $\Delta \cup \Delta_{P} \cup\left\{\left((s, n), \varepsilon, s^{\prime}\right) \mid s^{\prime} \in\right.$ matches $\left.[(s, n)]\right\}$, and $F_{2}=F$.

It is worth mentioning that the test performed at Line 6 can be carried out efficiently by a search in the transition graph of the automata. Details of an efficient implementation are available in [LAS].

A central step of the algorithm consists of refining the prefix automaton $\mathcal{A}_{P}$ in order to associate different end states to two sign headers $u$ and $u^{\prime}$ read 
from the state $s^{\prime}$ of $\mathcal{A}_{1}$. This operation is performed as follows:

(1) Let $k \in\{1, \ldots, n\}$ be the smallest integer such that the paths reading $u$ and $u^{\prime}$ from the state $s_{P}^{(0)}$ of $\mathcal{A}_{P}$ reach the same state after having followed $k$ transitions, and the paths reading $u$ and $u^{\prime}$ from the state $s^{\prime}$ of $\mathcal{A}_{1}$ reach two distinct states after the same number $k$ of transitions.

(2) Let $\left(\left(s_{1}, k-1\right), d,\left(s_{2}, k\right)\right)$ and $\left(\left(s_{1}^{\prime}, k-1\right), d^{\prime},\left(s_{2}, k\right)\right)$ be the $k$-th transitions of the paths reading (respectively) $u$ and $u^{\prime}$ in $\mathcal{A}_{P}$.

(3) For each $q \in Q_{P}$ such that $\left(\left(s_{2}, k\right), w, q\right) \in \Delta_{P}^{*}$ for some $w \in \Sigma^{*}$, add a new state $q^{\prime}$ to $Q_{P}$ and set $\operatorname{split}[q]=q^{\prime}$.

(4) For each transition $\left(q, d, q^{\prime}\right) \in \Delta_{P}$ such that split $[q]$ is defined, add the transition $\left(\operatorname{split}[q], d\right.$, split $\left.\left[q^{\prime}\right]\right)$ to $\Delta_{P}$.

(5) Replace the transition $\left(\left(s_{1}^{\prime}, k-1\right), d^{\prime},\left(s_{2}, k\right)\right)$ by $\left(\left(s_{1}^{\prime}, k-1\right), d^{\prime}, \operatorname{split}\left[\left(s_{2}, k\right)\right]\right)$ in $\Delta_{P}$.

(6) For each $q \in Q_{P}$ such that split $[q]$ exists, let matches $[$ split $[q]]=$ matches $[q]$.

(7) For each $\left(s, s^{\prime}\right) \in$ remaining such that split $[(s, n)]$ is defined, add the pair $\left(\operatorname{split}[(s, n)], s^{\prime}\right)$ to remaining.

\section{B Cycle classification and removal algorithm}

(1) Let $\mathcal{A}=\left(\Sigma, Q, s^{(0)}, \Delta, F\right)$, let visited $=\emptyset$, and for each state $s \in Q$, let leads-to-cycle $[s]=\mathbf{F}$

(2) If explore $\left(s^{(0)}, 0\right)=\mathbf{F}$, then the set represented by $\mathcal{A}$ is infinite. Otherwise, the automaton $\mathcal{A}^{\prime}$ is given by $\left(\Sigma, Q, s^{(0)}, \Delta, F\right)$.

Subroutine explore $(s, k)$ :

(1) Let visited $=$ visited $\cup\{s\}$, and let history $[k]=s$;

(2) For each $\left(s^{\prime}, d, s^{\prime \prime}\right) \in \Delta$ such that $s^{\prime}=s$ :

- If $s^{\prime \prime} \notin$ visited, then

(a) If explore $\left(s^{\prime \prime}, k+1\right)=\mathbf{F}$ then return $\mathbf{F}$;

(b) If leads-to-cycle $\left[s^{\prime \prime}\right]$ then let leads-to-cycle $[s]=\mathbf{T}$;

- If $s^{\prime \prime} \in$ visited and $(\exists i<k)\left(\right.$ history $\left.[i]=s^{\prime \prime}\right)$, then

(a) If leads-to-cycle[s] then return $\mathbf{F}$;

(b) Let leads-to-cycle $[s]=\mathbf{T}$, and remove $\left(s^{\prime}, d, s^{\prime \prime}\right)$ from $\Delta$;

- If $s^{\prime \prime} \in$ visited and $(\forall i<k)\left(\right.$ history $\left.[i] \neq s^{\prime \prime}\right)$, then

(a) If leads-to-cycle $\left[s^{\prime \prime}\right]$ then return $\mathbf{F}$;

(3) Return T. 